\title{
Three-Dimensional Vibration-Induced Vestibulo-ocular Reflex Identifies Vertical Semicircular Canal Dehiscence
}

\author{
Swee Tin Aw ${ }^{1,2}$, Grace Elizabeth Aw $^{2}$, Michael John Todd ${ }^{2}$, Andrew Philip Bradshaw $^{2}$, and Gabor \\ Michael Halmagyi ${ }^{1,2}$ \\ ${ }^{1}$ Central Clinical School, Faculty of Medicine, University of Sydney, Sydney, Australia \\ ${ }^{2}$ Institute of Chinical Neuroscience, Royal Prince Alfred Hospital, Camperdown, Sydney, NSW 2050, Australia
}

Received: 1 December 2010; Accepted: 11 May 2011; Online publication: 1 June 2011

\begin{abstract}
Vertical semicircular canal dehiscence (VSCD) due to superior canal dehiscence (SCD) or posterior canal dehiscence (PCD) of the temporal bone causes vestibular and cochlear hypersensitivity to sound. This study aimed to characterize the vibration-induced vestibulo-ocular reflex (ViVOR) in VSCD. ViVORs in one PCD and 17 SCD patients, confirmed by CT imaging reformatted in semicircular canal planes, were measured with dual-search coils as binocular three-dimensional eye rotations induced by skull vibrations from a bone oscillator (B71-10 ohms) at $7 \mathrm{~ms}, 500 \mathrm{~Hz}, 135-\mathrm{dB}$ peak-force level $(\mathrm{re}: 1 \mu \mathrm{N})$. The ViVOR eye rotation axes were computed by vector analysis and referenced to known semicircular canal planes. Onset latency of the ViVOR was $11 \mathrm{~ms}$. ViVOR from VSCD was up to nine times greater than normal. The ViVOR's torsional rotation was always contraversive-torsional (the eye's upper pole rotated away from the stimulated ear), i.e. its direction was clockwise from a left and counterclockwise from a right VSCD, thereby lateralizing the side of the VSCD. The ViVORs vertical component distinguishes PCD from SCD, being downwards in PCD and upwards in SCD. In unilateral VSCD, the ViVOR eye rotation axis aligned closest to the dehiscent vertical semicircular canal axis from either ipsilateral or contralateral mastoid vibrations. However, in bilateral VSCDs, the ViVOR eye rotation axis lateralized to the ipsilateral dehiscent
\end{abstract}

Correspondence to: Swee Tin Aw · Institute of Clinical Neuroscience . Royal Prince Alfred Hospital - Camperdown, Sydney, NSW 2050, Australia. Telephone: +612 9515 7592; fax: +612 9515 8347; email: sweea@icn.usyd.edu.au vertical semicircular canal axis. ViVOR was evoked in ossicular chain dysfunction, even when air-conducted click vestibulo-ocular reflex (VOR) was absent or markedly reduced. Hence, ViVOR could be a useful measurement to identify unilateral or bilateral VSCD even in the presence of ossicular chain dysfunction.

Keywords: vibration-induced vestibulo-ocular reflex, superior canal dehiscence, posterior canal dehiscence, ossicular chain dysfunction

\section{INTRODUCTION}

Vestibular and cochlear hypersensitivity to sound in the presence of a third labyrinthine window caused by a VSCD often presents with oscillopsia, vertigo, nystagmus induced by loud sounds and autophony, hyperacusis and apparent conductive hearing loss (Minor et al. 1998; Brantberg et al. 2006; Aw et al. 2006, 2010). VSCD can be an SCD due to a superior canal roof defect (Minor et al. 1998; Aw et al. 2006) or a PCD due to a high-riding jugular bulb (Aw et al. 2010) or an erosive middle ear cholesteotoma (Brantberg et al. 2006). Although high-resolution temporal bone computed tomography (CT) provides anatomical evidence of a semicircular canal dehiscence, it can overestimate the occurrence of the bony dehiscence (Williamson et al. 2003; Aw et al. 2006; Freidmann et al. 2010). Hence, measurement of vestibular hypersensitivity to air-conducted sound (Minor et al. 1998; Aw et al. 2006, 2010; Brantberg et al. 2006) or skull vibrations (Manzari et al. 2008; Welgampola et al. 2009) provides a functional diagnosis of a VSCD. However, in the presence of 
ossicular chain dysfunction, skull vibration may be useful as a diagnostic aid. However skull vibrations induced by a hand-held vibrator unpredictably induced either excitatory or inhibitory nystagmus in SCD (Manzari et al. 2008). Since skull vibrations are believed to be transmitted to both labyrinths, such vibrations may have difficulty identifying the location of the semicircular canal dehiscence. Although ViVOR has been reported in unilateral SCD (Welgampola et al. 2009), the following are not known: (1) ViVOR in PCD, (2) ViVOR patterns and magnitudes in bilateral VSCD when both dehiscent semicircular canals are hypersensitive and simultaneously activated during skull vibrations, and (3) whether ossicular chain integrity is required for eliciting the ViVOR. Using a repeatable stimulus from a bone oscillator (B71-10 ohms at $7 \mathrm{~ms}$, $500 \mathrm{~Hz}$ ), we showed that the excitatory and reproducible three-dimensional (3-D) ViVOR evoked by the bone oscillator not only identifies unilateral or bilateral VSCDs, it distinguishes PCD from SCD and it bypasses the ear's conductive mechanism.

\section{METHODS}

Subjects

ViVORs were recorded in 18 VSCD patients (range, 26-82 years; $52.2 \pm 14.1$ years, mean \pm 1 standard deviation) and compared with eight normal subjects. Seventeen (five bilateral, seven left and five right) SCD and one right PCD patients were diagnosed based on a combination of tests as shown in Table 1: (a) air-conducted single-click-evoked vestibulo-ocular reflex (click VOR) with magnitude and threshold below normal 110-dB normal hearing level (NHL; 145-dB sound pressure level) measurements (Aw et al. 2006); (b) high-resolution, 0.625-mm multi-slice (64), temporal bone CT images (GE Medical Systems, USA) reformatted in semicircular canal planes (Williamson et al. 2003; Aw et al 2006; Freidmann et al. 2010; Bradshaw et al. 2010) with estimation of the dehiscence arc and reconstruction in three dimensions, (c) threshold of cervical vestibular-evoked myogenic potentials from air-conducted single click (Rosengren et al. 2008), (d) clinical vestibular assessment such as pure tone $(0.25,0.50,1.0$, and $2.0 \mathrm{kHz})$ at $110-\mathrm{dB}$ NHL sound-induced nystagmus and pressure-induced nystagmus with the Hannebert and valsalva tests, (e) cochlear hypersensitivity symptoms such as autophonia/hyperacusis symptoms and pure-tone audiogram with crossed acoustic reflex. The patients' ViVORs were then compared with the above combination of tests elucidated in Table 1. The protocol was approved by our local Ethics Committee (Sydney South West Area Health Service Ethics Committee
Protocol no. X03-0063) in accordance with the Helsinki II Declaration.

\section{Case histories of patients}

Two SCDs (one unilateral (patient 11) and one bilateral (patient 3)) had left ossicular chain dysfunction after left partial ossicular chain replacement prosthesis (PORP) surgeries. Following a head injury, patient 11 with the unilateral SCD experienced pulsatile tinnitus in her left ear with severe autophonia during eye blinking, chewing, biting, walking and touching her head. She was misdiagnosed and had left PORP surgery with replacement of the incus, performed in 2004, which further exacerbated her symptoms. Despite a correct diagnosis of superior canal dehiscence syndrome, she elected not to have superior semicircular plugging (Fig. 3A). Patient 3 with bilateral SCD first had left PORP surgery in 1991 (prior to the first description of superior canal dehiscence syndrome by Minor et al. 1998) because of mild hearing loss and symptoms of cochlear hypersensitivity to sound such as hyperacusis, autophonia and pulsatile tinnitus. In the intervening years, because of fluctuating hearing loss, he had three left PORP surgical revisions with removal of the incus. During a bout of violent sneezing 7 years ago, he heard a loud "pop" in his left ear and immediately could not hear from that ear even with his hearing aid. Exploration of his middle ear was performed and subluxation of the PORP and severe necrosis of the stapes were noted and reattachment of the prosthesis was attempted. However, post-surgical CT confirmed that reattachment was unsuccessful and since then he had no further surgery (Fig. 3B).

The PCD patient complained oscillopsia following head injury, which he described as blurring of vision when listening to loud music or to the telephone with his right ear. He was diagnosed with air-conducted single-click VOR (Fig. 1B), together with five clicktrain-evoked vestibulo-ocular reflex, ocular-evoked vestibular myogenic potentials. Temporal bone CT with 3-D reconstruction in the posterior canal plane showed posterior canal dehiscence following trauma as a result of a right high-riding jugular bulb (Aw et al. 2010) and other tests shown in Table 1.

\section{Recording system}

Vibration-induced binocular, 3-D eye positions were recorded with precalibrated dual-search coils (Skalar, The Netherlands) in supine, head-fixed subjects and viewing a stationary target at $600 \mathrm{~mm}$ (Aw et al. 2006), with the subject's head in the centre of the transmitter field coils $\left(660 \mathrm{~mm}^{3}, 66 \mathrm{kHz}\right.$ and $100 \mathrm{kHz}, \mathrm{CNC}$ Engineering, USA). Search coil and vibration signals were sampled at $5 \mathrm{kHz}$ and 24-bit resolution (NI 


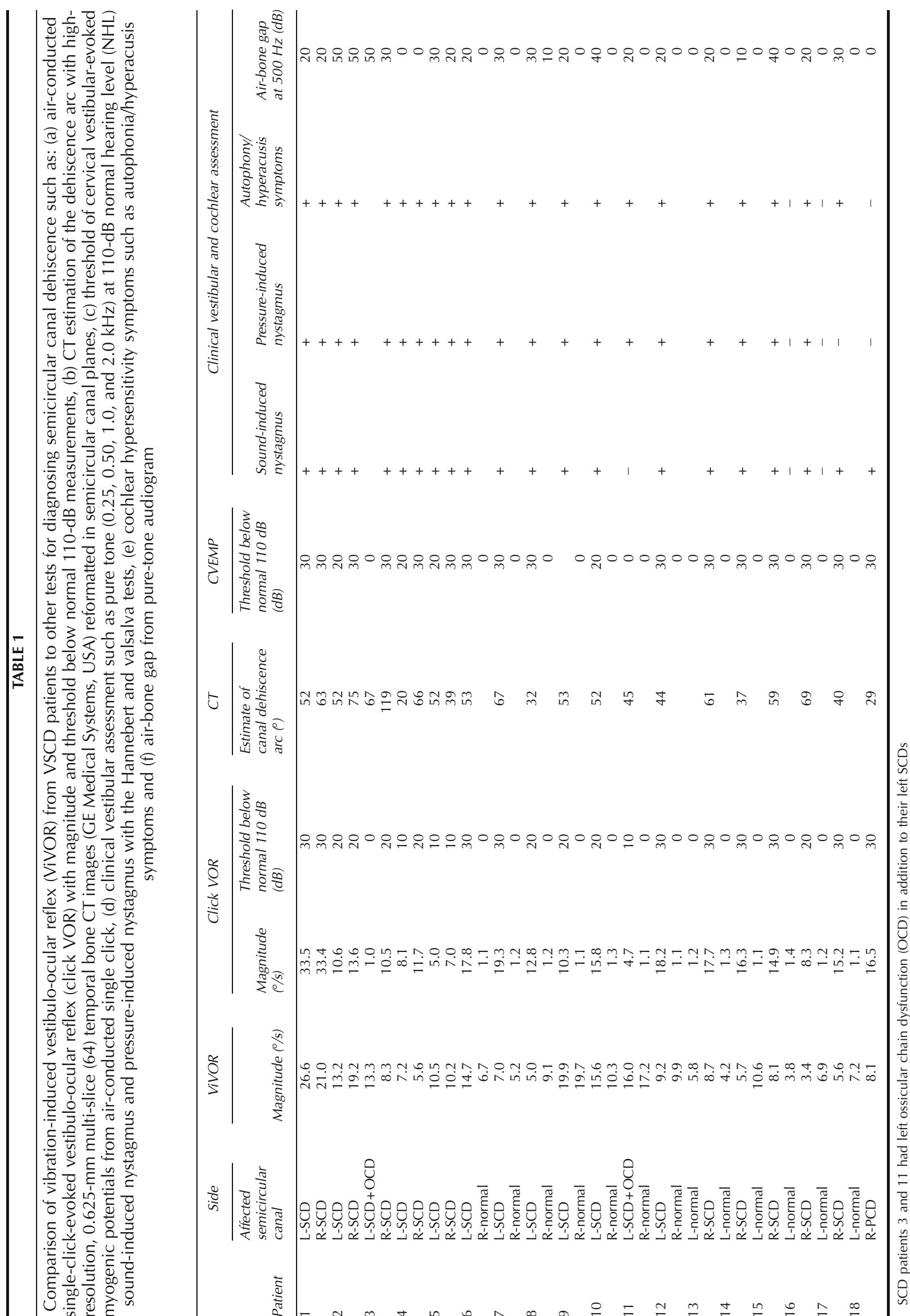


A $\begin{gathered}\text { Left SCD } \\ \text { Eye Position }\end{gathered}$ B $\begin{gathered}\text { Right PCD } \\ \text { Eye Position }\end{gathered}$
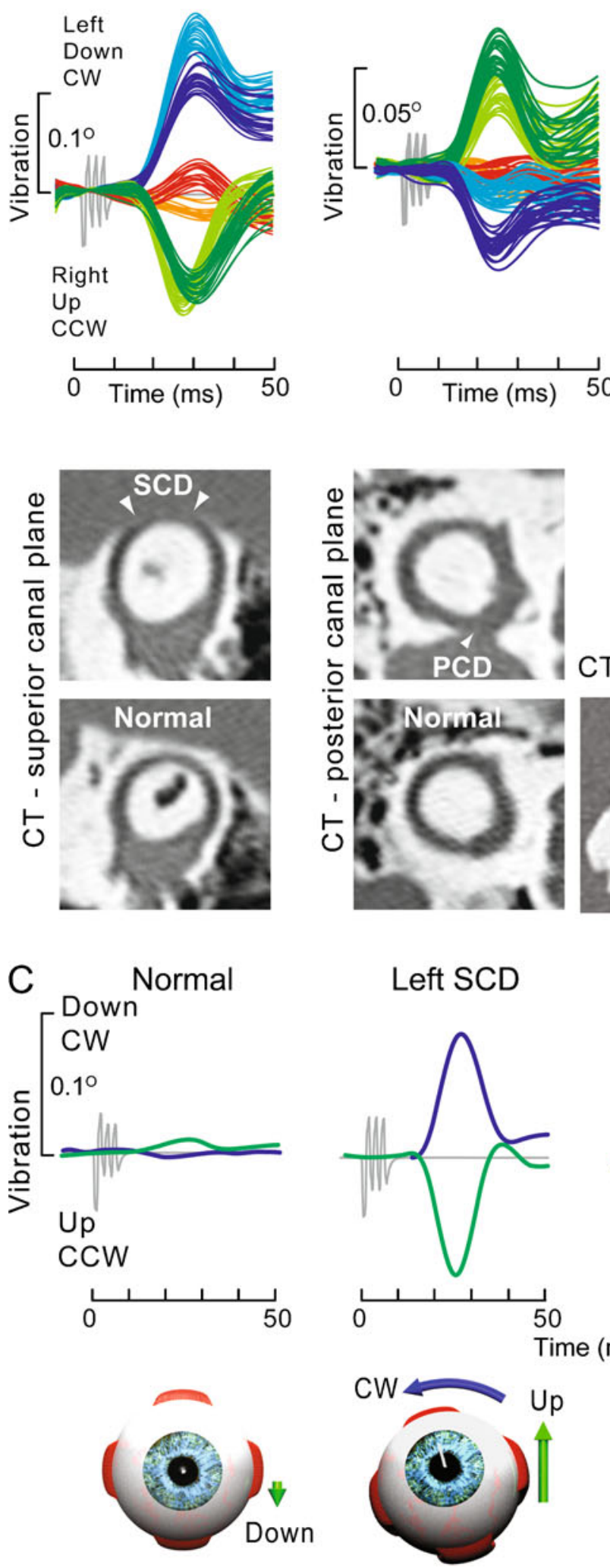
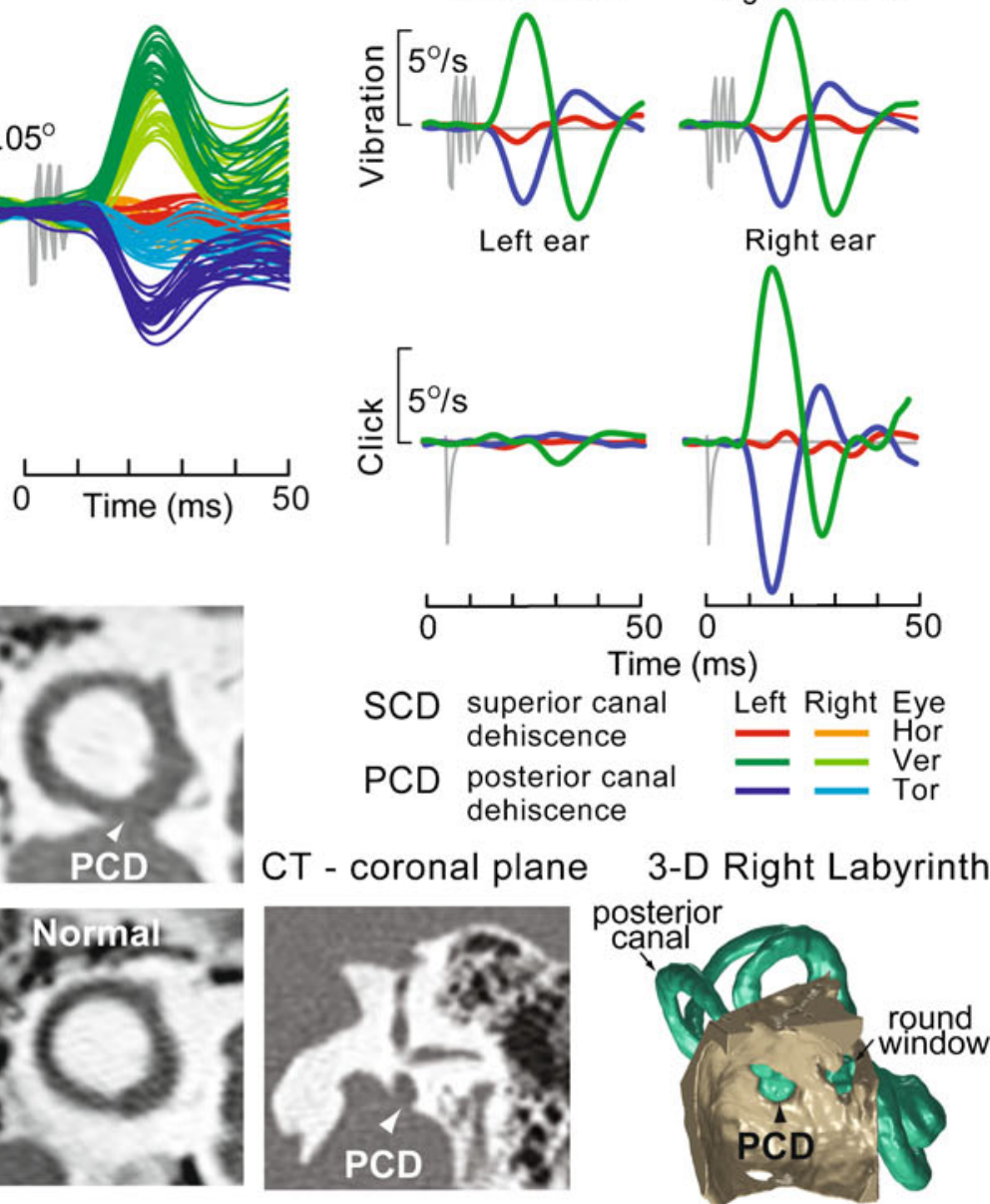

Right SCD
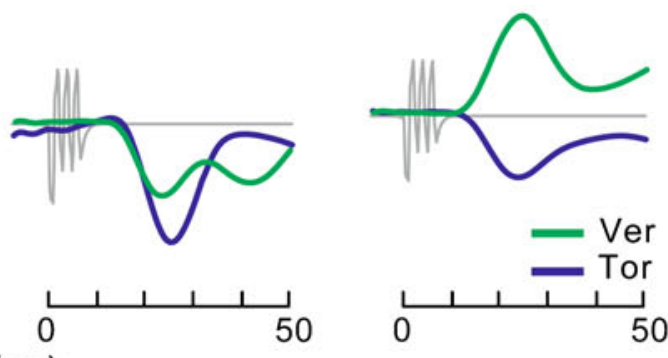

Time (ms)
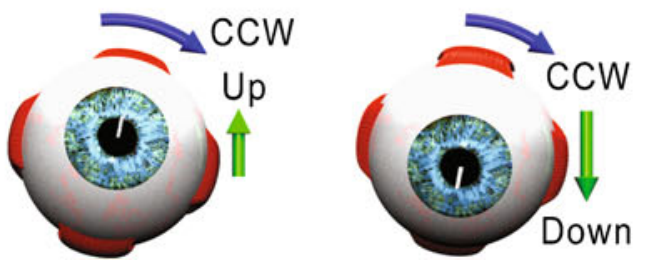
FIG. 1. ViVOR from VSCD patients confirmed by CT imaging. A Representative binocular, 3-D ViVOR eye position from a left superior canal dehiscence $(S C D)$ from multiple trials. The left SCD was confirmed by temporal bone $\mathrm{CT}$ images reformatted in superior canal plane in comparison to normal superior canal. B Representative binocular, 3-D ViVOR eye positions from a right posterior canal dehiscence $(P C D)$ from multiple trials. The ViVOR eye velocity from left mastoid and right mastoid bone-conducted vibrations were compared with air-conducted click VOR eye velocity in this right PCD patient. The right PCD was confirmed by temporal bone CT images reformatted in posterior canal plane in comparison to normal posterior canal, also shown in the coronal plane and reconstructed in three dimensions (3-D). C Normal ViVOR comprised miniscule downward eye rotations, but ViVOR from VSCD was enlarged, always contraversive-torsional (the eye's upper pole rotated away from the stimulated ear), upwards in SCD and downwards in PCD. CT images of the normal subject's superior canal and posterior canal were used for the above CT comparison. The ViVOR's torsional and vertical components can be used to identify the dehiscent semicircular canals as illustrated by the eyeball rotations.

PCI-4472, National Instruments, USA) with Labview (Version 7.1, National Instruments, USA). Resolution of the recording system was 0.1 arcmin for horizontal and vertical components and 0.3 arcmin for torsional component. Maximum errors and cross coupling were $<2 \%$.

\section{Vibration stimulus}

Skull vibration from a bone oscillator (B71-10 ohms, Radioear, USA) at $7 \mathrm{~ms}, 500 \mathrm{~Hz}, 135-\mathrm{dB}$ peak-force level (re: $1 \mu \mathrm{N})$ from $20 \mathrm{~V}$-pp output calibrated with an artificial mastoid (RETFL=58.0; Brüel \& Kjær Model 4930, Denmark) (Todd et al. 2007), was applied for 60 trials each to a subject's right and left mastoid bone. During each trial, the stimulus and 3-D eye rotations were measured for $1,000 \mathrm{~ms}$, commencing $100 \mathrm{~ms}$ before the presentation of the 7-ms skull vibration.

\section{Click stimulus}

Air-conducted $100-\mu$ s single click at $110-\mathrm{dB}$ NHL (145 dB SPL) stimulus was delivered for 30 trials each to the subjects' left or right ear by a matched pair of TDH-49 earphones (Telephonics Corporation, USA).

\section{Computed axial tomography imaging}

Three-dimensional reconstruction and multiplanar reformatting of the CT images with a second-order planar fit to the semicircular canal centreline permitted the bony capsule's integrity to be examined over its entire circumference (Bradshaw et al. 2010). 3-D reconstruction of the left labyrinths and ossicular chains were performed for the two SCD patients after left PORP surgeries (Fig. 3) and their CT images were reformatted in their left superior semicircular canal plane (Bradshaw et al. 2010).

\section{Data analysis}

Binocular 3-D eye positions as rotation vectors, Euler's angles and eye velocities were computed in spacefixed coordinates. Leftward, downward and clockwise from subject's view were positive for horizontal $(z)$, vertical $(y)$ and torsional $(x)$ eye rotations. Rotation of the eye's upper pole is clockwise towards subject's right and counterclockwise towards the left. Eye velocity is characterized by magnitude $\left(\sqrt{x^{2}+y^{2}+z^{2}}\right)$ and direction $(\vec{n})$ i.e. its instantaneous eye rotation axis (Aw et al. 2006). Each horizontal, vertical and torsional eye velocity component was normalized as a unit vector by dividing the individual eye velocity component by magnitude. Normalised ViVOR eye rotation axes were computed by vector analysis and plotted in 3-dimensions (Houdini, Side Effects, Canada) with averaged (mean \pm 1 standard deviation) semicircular canal axes (Aw et al. 2006; Bradshaw et al. 2010). ViVOR latency was the time difference between the stimulus and eye velocity onsets when the baseline signal derivative exceeded one standard deviation (Aw et al. 2006).

\section{Statistical analysis}

Means \pm 1 standard error of mean (1 SEM) from ViVOR trials without blinks were determined. The mean $(x, y, z)$ vectors were determined for normalized eye rotation axes (Aw et al. 2006). Student's $t$ test for differences between two independent means was used to compare eye rotation differences between normal and VSCD subjects. A significance level of $p=0.05$ was used in the statistical analysis.

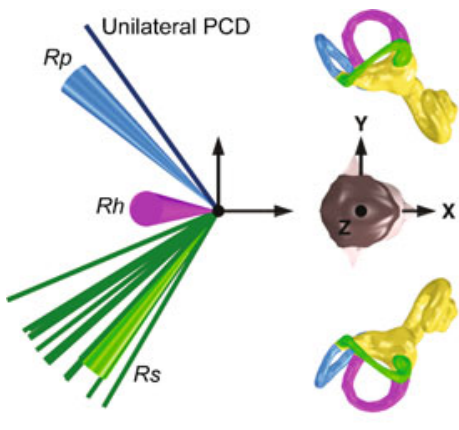

Unilateral SCDs

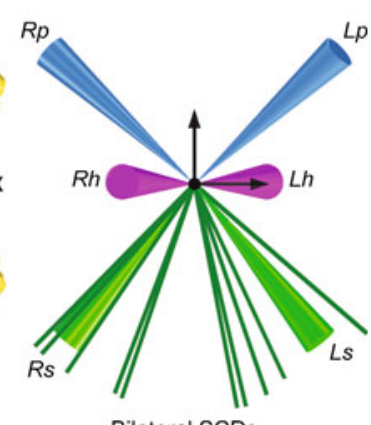

Bilateral SCDs
FIG. 2. ViVOR eye rotation axes from unilateral and bilateral VSCDs. The ViVOR eye rotation axes from 17 SCD (five bilateral, seven left and five right, with left pooled as right) and one right PCD by vector analysis and plotted in 3-D head-fixed coordinates $(x, y, z)$ were compared with right horizontal $(R h)$, superior $(R s)$, posterior $(R p)$ and left horizontal $(L h)$, superior $(L s)$ and posterior $(L p)$ averaged semicircular canal axes (cones). The eye rotation axes from unilateral and bilateral SCDs aligned closest to the superior semicircular canal axes and from unilateral PCD aligned closest to posterior semicircular canal axis. 
A

Unilateral Left SCD+OCD with Left PORP

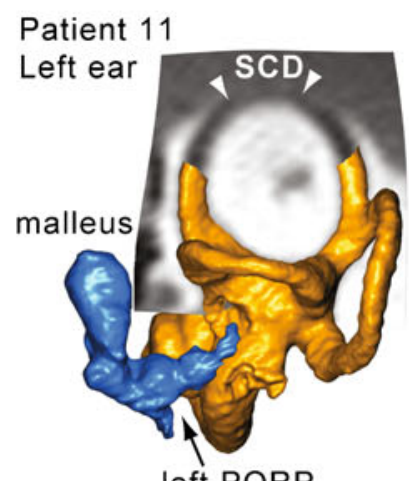

left PORP

Left mastoid Right mastoid

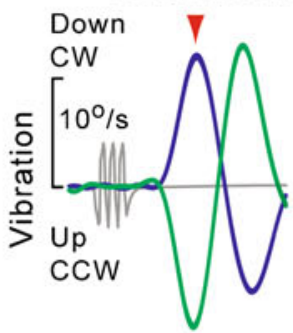

Left ear
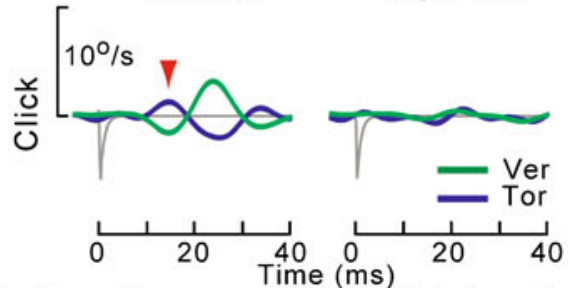

Axial CT

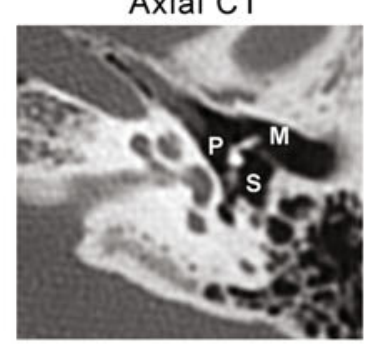

B
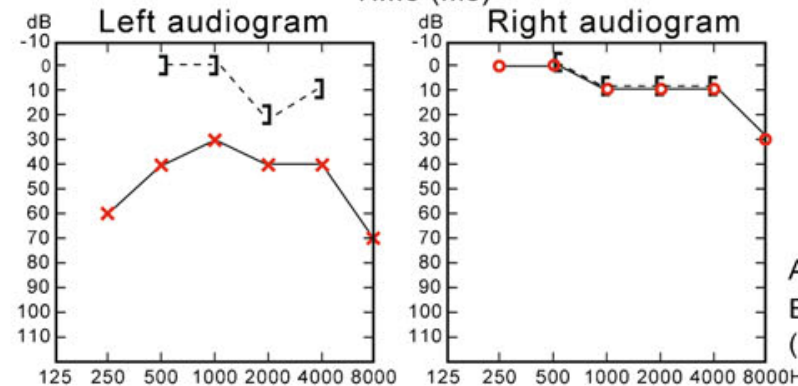

Bilateral SCD+OCD with Left PORP

Left mastoid

Right mastoid
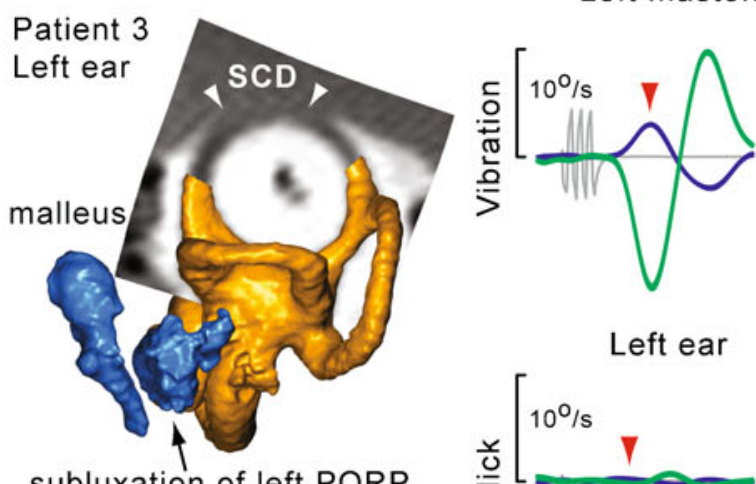

Left ear
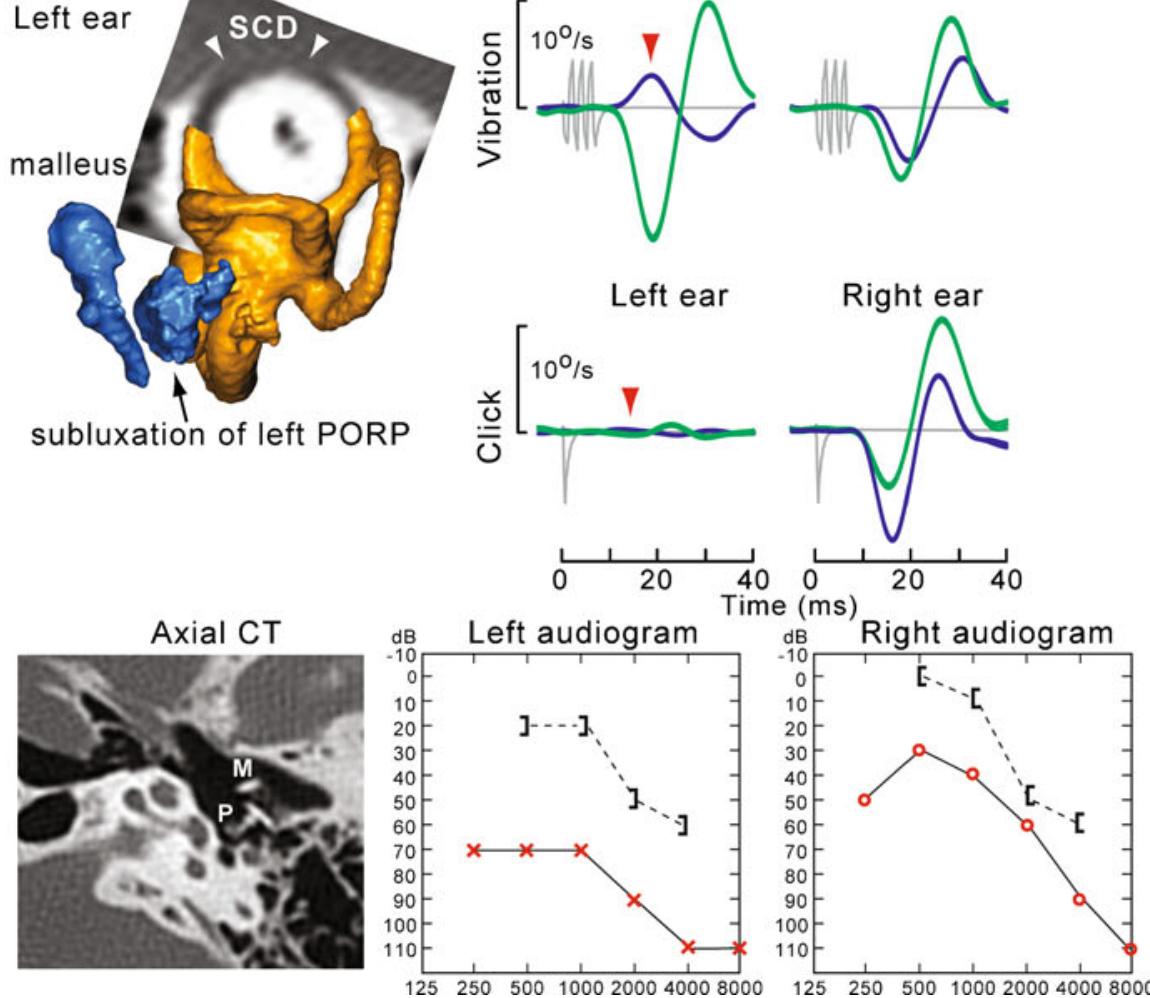

Unilateral Left SCD

(normal conductive mechanism)

Left mastoid Right mastoid

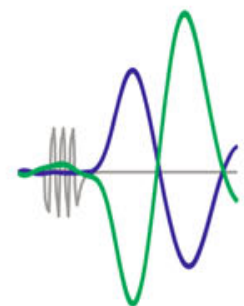

Left ear
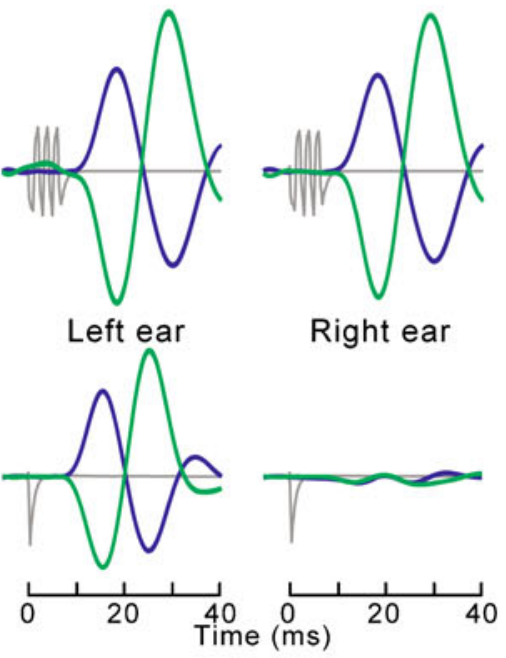

Right ear

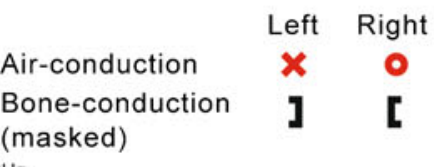

Bilateral SCD

(normal conductive mechanism) Left mastoid Right mastoid

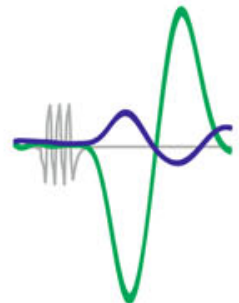

Left ear

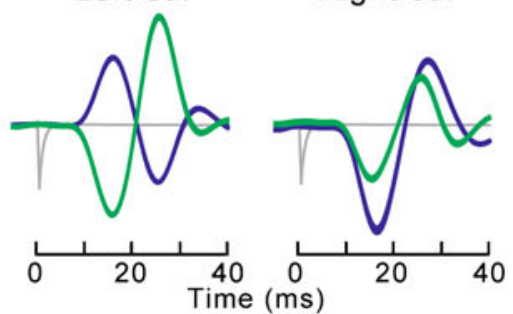

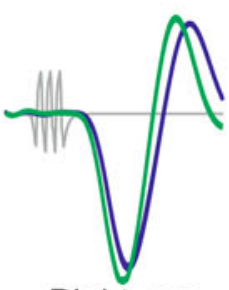

Right ear

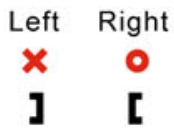


FIG. 3. ViVOR bypassed the ear's conductive mechanism and was preserved in ossicular chain dysfunction (OCD) after left partial ossicular chain replacement prosthesis (PORP) surgeries. A Comparison of the ViVOR to click VOR in two unilateral SCD patients. In the unilateral left SCD+OCD with left PORP (patient 11), ViVORs from left and right mastoid vibrations were preserved, but the left ear airconducted click VOR was significantly reduced (red arrow). In the unilateral left SCD with normal conductive mechanism, ViVOR was comparable to click VOR. B Comparison of the ViVOR to click VOR in two bilateral SCD patients. In bilateral SCD (patient 3), ViVORs from left and right mastoid vibrations were preserved, but click VOR was absent from the left SCD+OCD ear (red arrow) and enlarged from the right SCD ear. In the SCD patient with normal conductive mechanism, both ViVOR and click VOR were preserved. Axial CT image and 3-D reconstruction of the labyrinth shows the left PORP was articulating with malleus and stapes in the unilateral SCD, but the left PORP of the bilateral SCD was subluxed. (In the axial CT labelling $-M$ malleus, $P$ PORP, $S$ stapes.) Pure-tone audiograms for the unilateral and bilateral SCD patients after PORP surgeries were displayed.

\section{RESULTS}

\section{Enlarged ViVOR indicates vestibular} hypersensitivity

Normal ViVOR comprised miniscule downward eye rotations of amplitude $\left(y<0.01^{\circ}\right)$ (Fig. 1C) and eye velocity magnitude of $<2^{\circ} /$ s. Typical example of 3-D, binocular ViVOR eye positions $(x, y, z)$ from a left SCD comprised conjugate upward, clockwise eye rotations of the left eye $\left(x=0.16^{\circ}, y=-0.12^{\circ}, z=0.02^{\circ}\right)$ and right eye $\left(x=0.12^{\circ}, y=-0.10^{\circ}, z=0.02^{\circ}\right)$ and eye velocity magnitude of the left eye $=14.3^{\circ} / \mathrm{s}$; right eye $=19.9^{\circ} / \mathrm{s}$ from left mastoid vibrations (Fig. 1A). Horizontal eye rotations are usually small or absent. The left SCD was confirmed by temporal bone CT images reformatted in superior canal plane and compared with a normal superior canal.

Three-dimensional, binocular ViVOR eye positions from a right PCD comprised conjugate downward, counterclockwise with small or absent horizontal eye rotations of: left eye $\left(x=-0.04^{\circ}, y=0.05^{\circ}, z=0.00^{\circ}\right)$; right eye $\left(x=-0.02^{\circ}, y=0.04^{\circ}, z=0.01^{\circ}\right)$ from right mastoid vibrations (Fig. 1B). The PCDs bone-conducted ViVOR eye velocities from left and right mastoid vibrations were also compared with air-conducted click VOR eye velocities from the left and right ears. While enlarged ViVORs were induced by vibrating left mastoid $\left(\right.$ ViVOR magnitude $\left.=7.3^{\circ} / \mathrm{s}\right)$ and right mastoid (ViVOR magnitude $=8.1^{\circ} / \mathrm{s}$ ), enlarged click VOR (magnitude $=16.5^{\circ} / \mathrm{s}$ ) was induced only from his right ear. Right PCD was confirmed by temporal bone CT images reformatted in posterior canal plane and compared with a normal posterior canal; and also in coronal plane. Three-dimensional reconstruction of the PCD's right labyrinth showed the erosion of the posterior semicircular canal near the ampulla due a high-riding right jugular bulb (Fig. 1B).
Enlarged ViVOR showed vestibular hypersensitivity from unilateral or bilateral SCD and from unilateral PCD with enlarged amplitudes of up to $0.2^{\circ}$ and magnitudes up to $27^{\circ} / \mathrm{s} \quad(p<0.05)$ (Figs. 1 and 4 ). These ViVOR magnitudes from all SCD and PCD patients are shown in Table 1 and are also compared with the click VOR magnitudes and CT estimates of the dehiscence arc.

\section{ViVOR latency}

The ViVOR onset latency was $11.2 \pm 0.2 \mathrm{~ms}$ (mean \pm 1 SEM) for both eyes $(p>0.05)$.

\section{ViVOR identifies the VSCD}

The enlarged excitatory ViVOR's torsional and vertical components could identify the location of the VSCD (Fig. 1C). These patterns of the ViVOR's vertical and torsional components used to identify the dehiscent semicircular canals were illustrated in typical examples of normal, left and right SCDs and right PCD subjects. The ViVOR's torsional rotation was always contraversive-torsional (the eye's upper pole rotated away from the stimulated ear), i.e. its direction was clockwise from a left and counterclockwise from a right VSCD, thereby lateralizing the side of the VSCD. The ViVOR's vertical rotation was upwards in SCD and downwards in PCD thereby distinguishing SCD from PCD. The ViVOR's vertical $(y)$ and torsional $(x)$ components from normal was $\left(x=0.00^{\circ}, y=0.002^{\circ}\right)$, left SCD was $\left(x=0.09^{\circ}, y=\right.$ $\left.-0.09^{\circ}\right)$, right $\mathrm{SCD}$ was $\left(x=-0.04^{\circ}, y=-0.07^{\circ}\right)$ and right $\mathrm{PCD}$ was $\left(x=-0.04^{\circ}, y=0.05^{\circ}\right)$.

\section{ViVOR's eye rotation axis}

Skull vibrations were capable of simultaneously stimulating both labyrinths. In unilateral VSCD, ViVOR directions and magnitudes were similar $(p>0.05)$ from vibrations applied to the left or right mastoid bone (Fig. 2). The mean ViVOR eye rotation axis vector ( $x$, $y, z$ ) from 12 unilateral SCDs (left pooled as right) was $-0.74,-0.63$ and 0.02 and one right PCD was -0.58 , 0.81 and -0.09 . The ViVOR eye rotation axes in unilateral SCDs aligned closest to superior canal axis and in unilateral PCD it aligned closest to posterior canal axis. However, in bilateral VSCDs, the ViVOR eye rotation axis lateralized to the dehiscent vertical semicircular canal axis ipsilateral to the mastoid vibrations. ViVOR eye rotation axis from five bilateral SCDs was $0.37,-0.88$ and -0.03 from the left mastoid and $-0.52,-0.83$ and 0.04 from the right mastoid vibrations, which aligned closest to the superior canal axes ipsilateral to the vibrations. When bilateral SCDs vector $(-0.45,-0.85$ and 0.0 ; left pooled as right) was 
compared with unilateral SCDs vector $(-0.74,-0.63$ and 0.02 ), it showed a $27.7^{\circ}$ vector rotation from the $x$-axis towards the $y$-axis. This confirmed that although the ViVOR evoked from the labyrinth closest to applied vibrations predominated in bilateral VSCDs, there was some torsional cancellation and vertical summation again due to concurrent contralateral labyrinthine stimulation.

\section{Vibrations bypass the ear's conductive} mechanism

We verified that skull vibrations bypassed the ear's conductive mechanism by comparing the ViVORs from a unilateral and a bilateral SCD patient with left ossicular chain dysfunction with conductive hearing loss after left PORP surgeries (Fig. 3) with equivalent SCD patients with normal conductive mechanism. 3-D reconstruction and reformation of their CT images in the plane of their left superior semicircular canals were performed on these SCD patients' left labyrinths and ossicular chains to confirm their left SCDs and left PORPs (Bradshaw et al. 2010).

Figure 3A compares the ViVORs from the left unilateral SCD (patient 11) with left ossicular chain dysfunction (left panels) with the left unilateral SCD patient with normal conductive mechanism (right panels). The ViVOR eye velocities from these left SCDs comprised enlarged upward, contraversive-torsional, i.e. clockwise eye rotations from the left and right mastoid vibrations. In the left unilateral SCD with left ossicular chain dysfunction after left PORP surgery (left panels), the left mastoid ViVOR magnitude of $16.8^{\circ} \mathrm{s}$ was quadruple the markedly reduced left air-conducted click VOR magnitude of $4.7^{\circ} / \mathrm{s}(p<$ 0.05) (red arrows). Right mastoid ViVOR magnitude was $15.3^{\circ} / \mathrm{s}$ and the right click VOR was $1.1^{\circ} / \mathrm{s}$ from the normal right ear. However, in the left unilateral SCD with bilateral normal conductive mechanisms (right panels), left mastoid ViVOR magnitude of $15.6^{\circ} / \mathrm{s}$ was comparable to left ear air-conducted click VOR of $15.1^{\circ} / \mathrm{s} \quad(p>0.05)$. Right mastoid ViVOR magnitude was $15.3^{\circ} / \mathrm{s}$, but normal right ear click VOR was $1.1^{\circ} / \mathrm{s}$. These ViVOR eye rotation patterns (i.e. clockwise torsion and upwards) also showed that in unilateral VSCD, the ViVOR lateralized to the left dehiscent superior semicircular canal from vibrations of either left or right mastoid.

Figure 3B compares the ViVORs from a bilateral SCD patient (patient 3) with left ossicular chain discontinuity (left panels) with a bilateral SCD patient with normal conductive mechanism (right panels). The ViVOR eye velocities from these bilateral SCDs comprised enlarged upward and contraversive-torsional eye rotations, being clockwise from left mastoid and counterclockwise from right mastoid vibrations.
In the bilateral SCD with left prosthetic subluxation and ossicular chain discontinuity after a failed left PORP surgery (left panel), left mastoid ViVOR magnitude was $13.3^{\circ} / \mathrm{s}$, but left click VOR was absent $(p<0.05$; red arrows), and right mastoid ViVOR magnitude of $8.3^{\circ} / \mathrm{s}$ was comparable to right click VOR of $8.9^{\circ} / \mathrm{s}(p>0.05)$ because of the normal right conductive mechanism. However, in a bilateral SCD with normal bilateral conductive mechanisms (right panel), the left mastoid ViVOR magnitude of $14.2^{\circ} / \mathrm{s}$ and right mastoid ViVOR magnitude of $14.9^{\circ} / \mathrm{s}$ were comparable to the left click VOR of $11.2^{\circ} / \mathrm{s}$ and right click VOR of $13.4^{\circ} / \mathrm{s}$. Note that the patterns of the ViVOR eye rotations in bilateral VSCDs lateralized to the ipsilateral dehiscent superior semicircular canal exposed to the vibrations, i.e. the ViVOR was clockwise and upwards from the left mastoid vibrations but counterclockwise and upwards from right mastoid vibrations.

When we compared the ratio of ViVOR magnitude to Click VOR magnitude, the mean ratio for all patients was $1.46 \pm 1.09$ (group mean $\pm 95 \%$ confidence intervals). The left superior canal dehiscence with ossicular chain dysfunction $(\mathrm{SCD}+\mathrm{OCD})$ ratio from patient 11 was 13.3 and from patient 3 was 3.4, were different $(p<0.05)$ from patients with only SCD (Fig. 4A). Figure $4 \mathrm{~B}$ shows that there is no correlation between the ViVOR and the air-bone gaps of VSCD patients.

\section{Comparison of ViVOR from unilateral SCD to bilateral SCDs}

Figure 5 compares the ViVOR from unilateral SCD to ViVOR from bilateral left and right SCDs. In order to compare the ViVOR of different magnitudes (Table 1), individual ViVOR eye velocity components were normalized into unit vector (see data analysis). We showed that in unilateral left or right SCDs, the mean vertical component was approximately equal to torsional component. However, the mean vertical component was approximately double the torsional component in bilateral SCD suggesting that there was some torsional cancellation and vertical summation due to concurrent contralateral labyrinthine stimulation.

\section{DISCUSSION}

Normal vestibular organs are only slightly sensitive to sound and vibration (Aw et al. 2006; Todd et al. 2007; Welgampola et al. 2009). Our study again confirmed that normal subjects generated a miniscule downward ViVOR. However, ViVOR from VSCD was enlarged, up to nine times greater in 


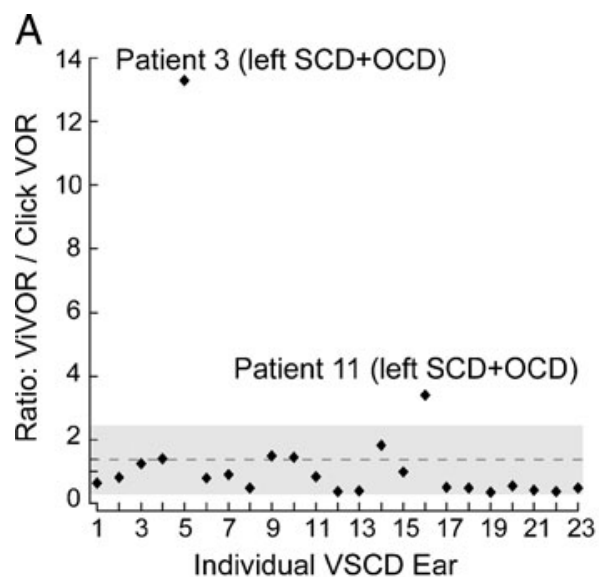

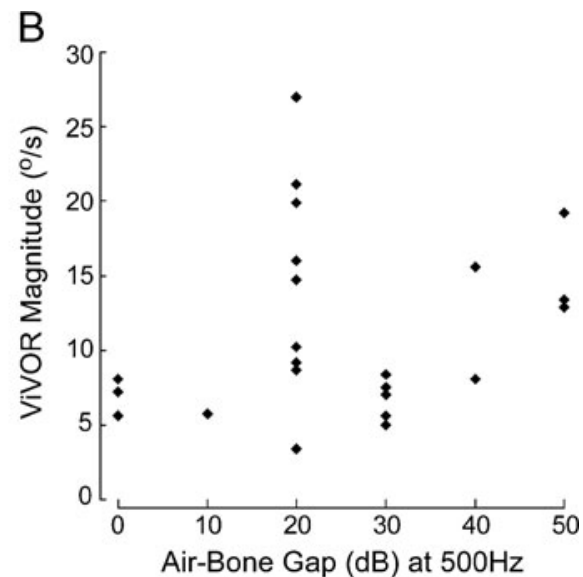

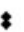

FIG. 4. A The ratio of bone-conducted ViVOR to air-conducted click VOR for individual SCD ear (diamonds) and group means $\pm 95 \%$ confidence intervals (grey) were displayed. Only the ears with SCD+ OCD were different. B There was no correlation between ViVOR magnitude and air-bone gap $(\mathrm{dB})$ of the VSCD. magnitude, substantiating vestibular hypersensitivity to skull vibration (Manzari et al. 2008; Welgampola et al. 2009). In our study, the ViVOR was always an excitatory response from the VSCD. We utilized this excitatory ViVOR's eye rotation directions to identify the VSCD based on the axiom from Ewald's first law (Ewald 1892) that the ViVOR eye rotation axis is orthogonal to the activated semicircular canal plane (Aw et al. 2005). The ViVORs torsional rotation was always contraversive-torsional (the eye's upper pole rotated away from the stimulated ear), i.e. its direction was clockwise from a left and counterclockwise from a right VSCD, thereby lateralizing the side of the VSCD (Fig. 1C). The ViVOR's vertical rotation was upwards in SCD (Aw et al. 2006; Welgampola et al. 2009) and downwards in PCD (Brantberg et al. 2006; Aw et al. 2010) hence distinguishing PCD from SCD.

Skull vibrations could simultaneously stimulate both labyrinths. In unilateral VSCD, compliance increase and vestibular hypersensitivity only from the dehiscent ear, coupled with insignificant ViVOR contribution from the normal labyrinth resulted in the eye rotation axes from ipsilateral or contralateral mastoid vibrations, aligning closest to the unilateral dehiscent vertical semicircular canal axis suggesting increase activation of the dehiscent semicircular canal receptors (Aw et al. 2006, 2010).

However in bilateral VSCDs, compliance increases and vestibular hypersensitivities were present in both labyrinths. The ViVOR eye rotation axis aligned closest to the dehiscent vertical semicircular canal axis ipsilateral to the vibrations, suggesting that the labyrinth closest to the vibrations received greater stimulation. Nonetheless in bilateral VSCD, the mean ViVOR eye rotation axis rotated $27.7^{\circ}$ from the $x$-axis towards the $y$-axis. This substantiated the presence of concurrent contralateral dehiscent vertical semicircular canal stimulation resulting in some torsional cancellation and vertical summation (Fig. 5B). This was because the torsional components from excitation of the left and right ears were in opposite directions, but vertical components were in the same direction because they are both SCD. However, unequal dehiscence sizes between the ears in bilateral VSCD could also influence the eye rotation directions.

The latency of ViVOR at about $11 \mathrm{~ms}$ was $2 \mathrm{~ms}$ longer than latency of air-conducted click VOR of $9 \mathrm{~ms}$ (Aw et al. 2006).

The advantage of our ViVOR generated from $7 \mathrm{~ms}, 500 \mathrm{~Hz}, 135-\mathrm{dB}$ peak-force-level mastoid vibrations was that the ViVOR was reproducible
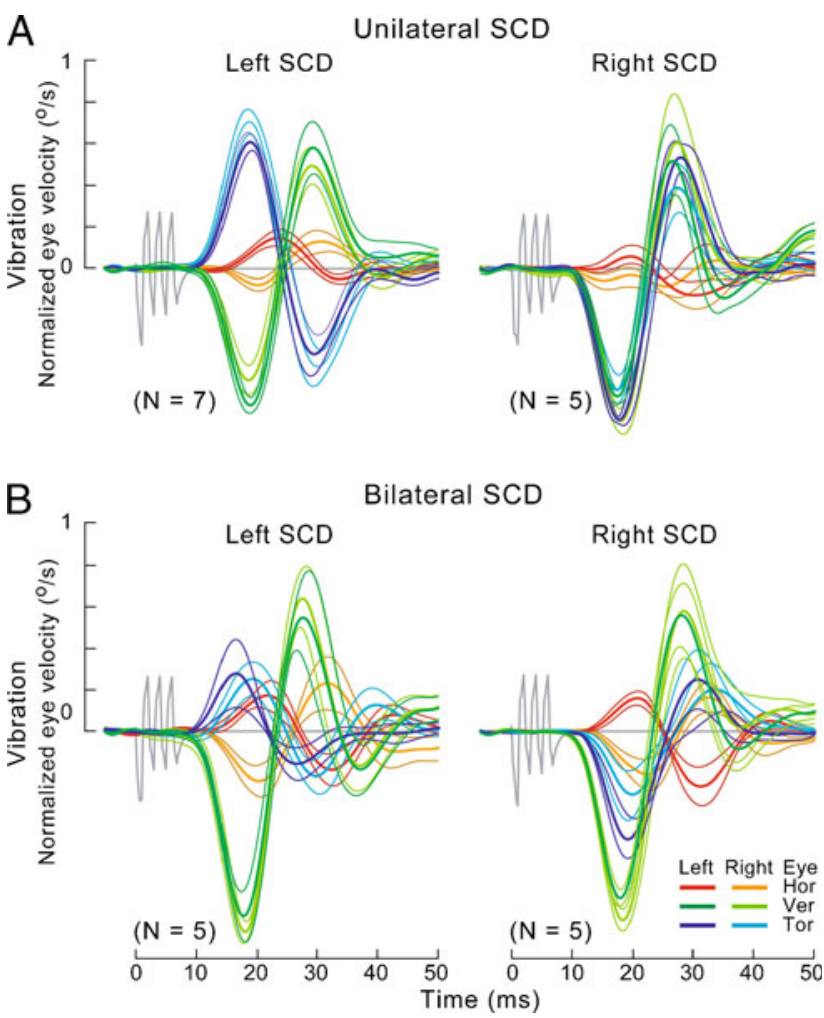

FIG. 5. Comparison of the group means \pm 1 standard error of the normalized, binocular, 3-D ViVOR eye velocities from seven left and five right unilateral superior canal dehiscence $(S C D)$ with five bilateral SCD patients. 
and always excitatory. In contrast, nystagmus induced by $2 \mathrm{~s}, 100 \mathrm{~Hz}$ vibrations from a hand-held vibrator could either be excitatory or inhibitory and, therefore, could not lateralize the dehiscent side nor distinguish PCD from SCD (Manzari et al. 2008). This is because excitatory ViVOR would be in the opposite directions to inhibitory ViVOR and not being able to reliably predict whether the response would be excitatory or inhibitory would hamper the usefulness of relying on nystagmus eye rotation directions to identify the VSCD based on Ewald's first law (Ewald 1892).

We confirmed that skull vibration did not rely on ossicular chain displacement (Irvine et al. 1979) for labyrinthine stimulation. This was corroborated by the bilateral SCD patient with the left PORP subluxation and ossicular chain discontinuity, where left mastoid bone-conducted ViVOR was enlarged and its eye rotation axis aligned closest to the left superior canal axis but air-conducted left click VOR was absent. Here, we verified that skull vibration bypassed the ear's conductive mechanism by directly activating the labyrinths to generate a ViVOR in the presence of ossicular chain discontinuity and therefore could be a useful diagnostic aid for detecting VSCD in patients with ossicular chain dysfunction.

Although ocular vestibular-evoked myogenic potentials induced by air-conducted sound showed vestibular hypersensitivity in SCD (Welgampola et al. 2009) and PCD (Aw et al. 2010), the responses cannot distinguish SCD from PCD. Air-conducted stimulus would also be required to lateralize the side of the VSCD, which would not be feasible in ossicular chain dysfunction. We showed that monocular ViVOR torsional and vertical components (as illustrated in Figs. 1C and 3) were sufficient to identify the VSCD as these components were conjugate (Fig. 1A). Even though the spatio-temporal resolution of the ViVOR recorded here is higher than the current real-time sampling frequency of $304 \mathrm{~Hz}$ capability of 3-D video-oculography (Schulz et al. 2011), however with technological advances, these ViVORs might in future be detectable by video-oculography for clinical diagnostic purpose. In addition, the ViVOR eye rotation amplitude in VSCD could be further enhanced by employing temporal summation techniques similar to clicktrains previously shown to enhance the click evoked VOR in SCD by two and half times (Aw et al. 2006).

\section{ACKNOWLEDGEMENTS}

The authors would like to thank all patients and control subjects for participating in the study. This research was funded by the National Health and Medical Research Council Australia, University of Sydney, Royal Prince Alfred Hospital Neurology Trustees and The Ramaciotti Foundation.

\section{Conflicts of interest}

The authors declare that they have no conflict of interest. Prof. GM Halmagyi is a consultant for GN Otometrics.

\section{REFERENCES}

Aw ST, Todd MJ, Aw GE, McGarvie LA, Halmagy GM (2005) Benign positional nystagmus: a study of its three-dimensional spatiotemporal characteristics. Neurology 64:1897-1905

Aw ST, Todd MJ, Aw GE, Magnussen JS, Curthoys IS, Halmagy GM (2006) Click-evoked vestibulo-ocular reflex: stimulus-response properties in superior canal dehiscence. Neurology 66:10791087

Aw ST, Welgampola MS, Bradshaw AP, Todd MJ, Magnussen JS, Halmagy GM (2010) Click-evoked vestibulo-ocular reflex distinguishes posterior from superior canal dehiscence. Neurology 75:933-935

Bradshaw AP, Curthoys IS, Todd MJ, Magnussen JS, Taubman DS, Aw ST, Halmagy GM (2010) A mathematical model of human semicircular canal geometry: a new basis for interpreting vestibular physiology. J Assoc Res Otolaryngol 11:145-159

Brantberg K, Bagger-Sjoback D, Mathiesen T, Witt H, Pansell T (2006) Posterior canal dehiscence syndrome caused by an apex cholesteatoma. Otol Neurotol 27:531-534

Ewald JMR (1892) Physiologische Untersuchungen über das Endorgan des Nervus octavus. Bergmann, Wiesbaden

Freidmann DR, Le BT, Pramanik BK, Lalwani AK (2010) Clinical spectrum of patients with erosion of the inner ear by jugular bulb abnormalities. Laryngoscope 120:365-372

Irvine DRF, Yates GK, Johnstone BM (1979) Bone conduction mechanisms: Mössbauer measurements on the role of ossicular inertia. Hear Res 1:101-109

Manzari L, Modugno GC, Brandolini C, Pirodda A (2008) Bone vibration-induced nystagmus is useful in diagnosing superior semicircular canal dehiscence. Audiol Neurootol 13:379-387

Minor LB, Solomon D, Zinreich JS, Zee DS (1998) Sound- and/or pressure-induced vertigo due to bone dehiscence of the superior semicircular canal. Arch Otolaryngol Head Neck Surg 124:249258

Rosengren SM, Aw ST, Halmagy GM, Todd NP, Colebatch JG (2008) Ocular vestibular evoked myogenic potentials in superior canal dehiscence. J Neurol Neurosurg Psychiatry 79:559-568

Schulz CM, Schneider E, Fritz L, Vockeroth J, Hapfelmeier A, Brandt T, Kochs EF, Schneider G (2011) Visual attention of anaesthetists during simulated critical incidents. Br J Anaesth (in press)

Todd NP, Rosengren SM, Aw ST, Colebatch JG (2007) Ocular vestibular evoked myogenic potentials (OVEMPs) produced by air- and bone-conducted sound. Clin Neurophysiol 118:381-390

Welgampola MS, Migliaccio AA, Myrie OA, Minor LB, Carey JP (2009) The human sound-evoked vestibulo-ocular reflex and its electromyographic correlate. Clin Neurophysiol 120:158166

Williamson RA, Vrabec JT, Coker NJ, Sandlin M (2003) Coronal computed tomography prevalence of superior semicircular canal dehiscence. Otolaryngol Head Neck Surg 129:481-489 